\title{
Low-Complexity and Robust Quantized Hybrid Beamforming and Channel Estimation
}

\author{
Sohail Payami, Christos Masouros*, Mathini Sellathurai \\ Heriot-Watt University, ${ }^{*}$ University College London, UK \\ Emails: \{s.payami, m.sellathurai\}@hw.ac.uk \\ , *c.masouros@ucl.ac.uk
}

\begin{abstract}
Hybrid beamforming with phase shifters and switches has been identified as a low-cost and energy-efficient approach to harness the benefits of massive multiple-input multipleoutput (MIMO) systems. In this paper, three subconnected hybrid beamforming structures with different combinations of phase shifters and switches will be considered. Firstly we assume that perfect channel state information (CSI) is available and the wireless channel follows uncorrelated Rayleigh fading model. Then, we derive the closed-form expressions of the low-complexity beamformers and their asymptotic achievable sum-rates. Based on the proposed beamformers, we develop quantized hybrid beamforming and channel estimation techniques for correlated Rayleigh fading channels. These methods rely on designing novel RF codebooks and they can be used in both CSI acquisition and data transmission phases. The proposed methods benefit from low computational complexity, low signaling overhead and robustness to estimation errors. Moreover, they are applicable to both frequency and time division duplex systems.
\end{abstract}

Index Terms-Massive MIMO, hybrid beamforming, phase shifter selection, quantized beamforming.

\section{INTRODUCTION}

Fully-digital beamforming in massive multiple-input multiple-output (MIMO) technology can significantly scale up the spectral efficiency in cellular systems [1]-[3]. However, it is an expensive technology and also suffers from low energy efficiency because it requires a dedicated power-hungry and expensive radio frequency (RF) chain per antenna [4]. One of the promising alternatives to exploit the benefits of massive MIMO with lower cost and better energy efficiency is hybrid analog-and-digital beamforming. In this approach, a small number of RF chains are connected to a large number of antennas through a fully-connected or subconnected network of phase shifters and/or switches [4]-[11].

In the fully-connected structure, each RF chain is connected to all the antennas as in [5]-[11]. Although it can exploit the full array gain, its power consumption can be very high due to the massive number of the phase shifters in the RF network [6], [12]. Moreover, the large number of the required RF routes results in fabrication complexity and high crosstalk distortion. Hence, the subconnected structure, where each RF chain is connected to a subset of antennas, is preferred in practice. In this direction, the focus of this paper is also on subconnected structure.

At the RF beamformer, switches offer lower power consumption compared to phase shifters at the cost of lower spectral efficiency. In order to provide a tradeoff, recently we proposed the concept of phase shifter selection which can significantly improve the energy efficiency while the achievable sum-rate is almost preserved [6]-[8]. Phase shifter selection allows for identifying and turning off the phase shifters that have a smaller contribution to spectral efficiency. In addition, it allows for scaling up the array size at the base station with just inexpensive antennas and switches [7], [8].

Designing hybrid beamformers is a challenging task due to the nonconvex constraints that are imposed by phase shifters and switches. Consider a base station which is equipped with a large number of antennas $N$ and it serves a smaller number of users $K$. The computational complexity of most of the existing algorithms is at least $O\left(K N^{2}\right)$ [6]. Under perfect channel state information (CSI) assumption, [13] showed that using equal gain transmission in hybrid beamformers, with only phase shifters, can achieve a near-optimal performance in frequencyflat channels. This approach reduces the computational complexity of the hybrid beamformer to $O\left(K^{3}\right)$.

With this motivation, it is desirable to benefit from the lowcost and energy-efficient phase shifter selection based structures of [7], [8], and the low-computational complexity of [13]. However, a common challenge for [7], [8], [13] is that these works rely on the availability of perfect CSI at the base station which is not feasible in practice. Moreover, channel estimation for massive MIMO systems with hybrid beamfomers requires a large signaling overhead. In this direction, we are aiming to pursue two objectives in this paper:

- Assuming the availability of perfect CSI of the uncorrelated Rayleigh fading channel, we propose low-complexity phase shifter selection based hybrid beamforming techniques which only require $O\left(K^{3}\right)$ instead of $O\left(K N^{2}\right)$ in our previous work [6]-[8]. In addition, the closed-form expressions of both hybrid beamformers and their asymptotic performance will be presented. This can be used as a design guide to evaluate the approximate performance of the proposed methods.

- In order to consider a more realistic scenario, we present simple and low-complexity quantized hybrid beamforming and channel estimation techniques which are designed according to the second order statistics of the correlated Rayleigh fading channel. The advantages of the proposed techniques are: a) The complexity of the beamformer is related to $O\left(K^{3}\right)$; b) The channel estimation overhead 
mainly depends on the rank of the correlation matrix of the channel; Hence, c) just by using low-cost switches and without increasing the signaling overhead to estimate the channel, a low cost and complexity massive MIMO can be deployed; d) The simulation results indicate that the proposed methods are more robust to estimation errors compared to the scenario that hybrid beamformer is designed based on the estimated propagation channel.

\section{SYSTEM MODEL}

Consider a single-cell multiuser massive MIMO downlink scenario where the base station has $N$ omni-directional antennas and serves $K \ll N$ single-antenna users. Base station applies $\mathbf{F} \in \mathbb{C}^{N \times K}$ to precode the vector of modulated symbols $\mathbf{u} \in \mathbb{C}^{K \times 1}$ with $\mathrm{E}\left[\mathbf{u u}^{\mathrm{H}}\right]=\mathbf{I}_{K}$. The channel input vector is $\mathbf{x}=(\sqrt{\rho / \Gamma}) \mathbf{F u}$ where $\rho$ is a measure of signal-to-noise ratio (SNR) and $\Gamma=\operatorname{trace}\left(\mathbf{F F}^{\mathrm{H}}\right) / K$ is a transmit power normalization factor. The received signal vector $\mathbf{y} \in \mathbb{C}^{K \times 1}$ is $\mathbf{y}=\mathbf{H}^{\mathrm{H}} \mathbf{x}+\mathbf{z}$ where $\mathbf{H}^{\mathrm{H}} \in \mathbb{C}^{K \times N}$ and $\mathbf{z} \in \mathbb{C}^{K \times 1}$ represent downlink channel matrix and noise vector. The elements of normalized noise vector $\mathbf{z}$ follow independent and identically distributed (i.i.d.) Gaussian distribution according to $z_{k} \sim \mathcal{C N}(0,1)$. In this paper, we consider spectral efficiency as the performance metric. When fully-digital ZF precoder is used, the achievable sum-rate is [6]

$$
R=K \log _{2}\left(1+\frac{\rho}{\Gamma}\right) .
$$

Three hybrid beamforming structures, as shown in Fig. 1, will be investigated in this paper. Figure 1(a) shows the block diagram of subconnected structure with phase shifters only. In Fig. 1(b), each phase shifter is equipped with a switch to reduce the power consumption at the RF beamforming network by turning off some of the phase shifters. Figure 1(c) uses 1-outof- $S$ switches to reduce the number of the phase shifters when $N$ is fixed. In order to maximize the spatial multiplexing gain, we assume that the number of the RF chains is equal to the number of users $K$ [6]. In Fig. 1, $L$ is the number of phase shifters that are connected to each RF chain.

Let $\mathbf{F}^{(q)}, q \in\{$ SP, SPSS, SRPS $\}$ denote the precoding matrix for the hybrid beamforming structures with subconnected phase shifters (SP), subconnected with phase shifter selection (SPSS), and subconnected with reduced number of phase shifters (SRPS), respectively. In hybrid beamforming, the precoding matrix $\mathbf{F}^{(q)}$ can be decomposed as $\mathbf{F}^{(q)}=\mathbf{F}_{\mathrm{RF}}^{(q)} \mathbf{F}_{\mathrm{B}}^{(q)}$ where $\mathbf{F}_{\mathrm{RF}}^{(q)} \in \mathbb{C}^{N \times K}$ and $\mathbf{F}_{\mathrm{B}}^{(q)} \in \mathbb{C}^{K \times K}$ represent the RF and digital beamforming matrices. In the rest of this paper, the baseband precoder is always designed according to $\mathrm{ZF}$ over the effective channel such that $\mathbf{F}_{\mathrm{B}}^{(q)}=\left(\mathbf{H}_{\mathrm{e}}^{(q)}\right)^{-1}$ where $\mathbf{H}_{\mathrm{e}}^{(q)}=\mathbf{H}^{\mathrm{H}} \mathbf{F}_{\mathrm{RF}}^{(q)}$. The design of $\mathbf{F}_{\mathrm{RF}}^{(q)}$ according to the different CSI assumptions will be discussed in the following sections.

\section{Perfect CSI ANd UnCorrelated Rayleigh FADING CHANNELS}

In this section, we present hybrid beamforming methods for the structures in Fig. 1 with complexity $O\left(K^{3}\right)$. Let the channel

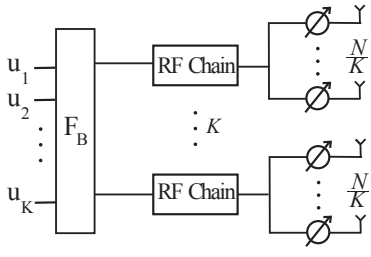

(a)

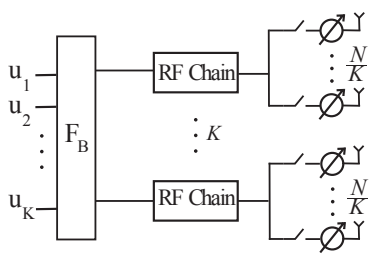

(b)

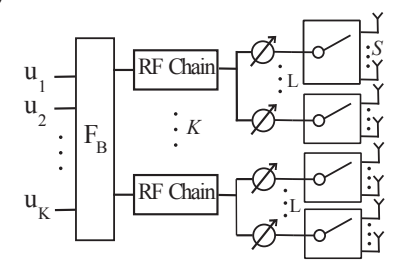

(c)

Fig. 1. a) Subconnected phase shifters (SP), b) subconnected with phase shifter selection (SPSS), c) subconnected with reduced number of phase shifters (SRPS).

coefficients $H_{n, k}$ follow frequency-flat, uncorrelated and i.i.d. Rayleigh fading model according to $H_{n, k} \sim \mathcal{C N}(0,1), \forall k \in$ $\{1, \ldots, K\}$ and $\forall n \in\{1, \ldots, N\}$. For Fig. 1(a) with analog phase shifters, $\mathbf{F}_{\mathrm{RF}}^{(\mathrm{SP})}$ is a block diagonal matrix such that $F_{\mathrm{RF}, n, k}^{(\mathrm{SP})}=$ $\mathrm{e}^{j \theta_{n, k}} / \sqrt{L}, \forall \theta_{n, k} \in[0,2 \pi)$ and $\forall n \in \mathcal{I}_{k}$ where $\mathcal{I}_{k}=\left\{\frac{N}{K}(k-\right.$ 1) $\left.+1, \ldots, \frac{N}{K} k\right\}$ and $L=N / K$, otherwise $\left|F_{\mathrm{RF}, \mathrm{n}, \mathrm{k}}^{(\mathrm{SP})}\right|=0, n \notin$ $\mathcal{I}_{k}$. Instead of designing $\mathbf{F}_{\mathrm{RF}}^{(\mathrm{SP})}$ according to the first $K$ left singular vectors of $\mathbf{H}^{\mathrm{H}}$, which requires $O\left(K N^{2}\right)$ [7], set the RF beamformer as

$$
F_{\mathrm{RF}, n, k}^{(\mathrm{SP})}= \begin{cases}\sqrt{\frac{1}{L}} \mathrm{e}^{j \angle H_{n, k}} & \text { if } n \in \mathcal{I}_{k}, \\ 0 & \text { if } n \notin \mathcal{I}_{k} .\end{cases}
$$

To derive the performance of the beamformer, let $\hat{\mathbf{h}}_{k}=$ $\mathbf{h}_{k} /\left\|\mathbf{h}_{k}\right\|$ denote the unit-norm vector in the direction of $\mathbf{h}_{k}$. The law of large numbers implies that $\left\|\mathbf{h}_{k}\right\| \rightarrow \sqrt{N}$ and the elements of $\hat{\mathbf{h}}_{k}$ are distributed according to $\hat{H}_{n, k} \sim \mathcal{C N}(0,1 / N)$, and hence $\mathrm{E}\left[\left|\sqrt{N} \hat{H}_{n, k}\right|\right]=\frac{\sqrt{\pi}}{2}$. Since the cardinality of $\mathcal{I}_{k}$ is $L=N / K$, using the law of large numbers results in

$$
\begin{aligned}
\hat{\mathbf{h}}_{k}^{\mathrm{H}} \mathbf{f}_{\mathrm{RF}, \mathrm{K}}^{\mathrm{SP})} & =\sqrt{\frac{K}{N}} \sum_{\forall n \in \mathcal{I}_{k}}\left|\hat{H}_{n, k}\right|=\frac{1}{\sqrt{K}}\left(\frac{K}{N} \sum_{\forall n \in \mathcal{I}_{k}}\left|\sqrt{N} \hat{H}_{n, k}\right|\right) \\
& \rightarrow \frac{\mathrm{E}\left[\left|\sqrt{N} \hat{H}_{n, k}\right|\right]}{\sqrt{K}}=\frac{\sqrt{\pi}}{2 \sqrt{K}} .
\end{aligned}
$$

Similarly, it could be shown that $\hat{\mathbf{h}}_{k^{\prime}}^{\mathrm{H}} \mathbf{f}_{\mathrm{RF}, k}^{(\mathrm{SP})} \rightarrow 0, \forall k^{\prime} \neq k$ as $\mathrm{E}\left[\sqrt{N} \hat{H}_{n, k}\right]=0$. In this case, $\mathbf{H}_{\mathrm{e}}^{(\mathrm{SP})}=\mathbf{H}^{\mathrm{H}} \mathbf{F}_{\mathrm{RF}}^{(\mathrm{SP})} \rightarrow$ $\sqrt{N \pi} /(2 \sqrt{K}) \mathbf{I}_{K}$. When ZF is applied at the baseband, the power normalization factor becomes

$$
\begin{aligned}
& \Gamma^{(\mathrm{SP})}=\operatorname{trace}\left(\mathbf{F}_{\mathrm{B}}^{(\mathrm{SP})^{\mathrm{H}}} \mathbf{F}_{\mathrm{RF}}^{(\mathrm{SP})^{\mathrm{H}}} \mathbf{F}_{\mathrm{RF}}^{(\mathrm{SP})} \mathbf{F}_{\mathrm{B}}^{(\mathrm{SP})}\right) / K \\
& \stackrel{(a)}{=} \operatorname{trace}\left(\left(\mathbf{H}_{\mathrm{e}}^{(\mathrm{SP})}\right)^{-1}\left(\mathbf{H}_{\mathrm{e}}^{(\mathrm{SP})}\right)^{-1}\right) / K \\
& \rightarrow \operatorname{trace}\left(\left(\frac{\sqrt{N \pi}}{2 \sqrt{K}} \mathbf{I}_{K}\right)^{-1}\left(\frac{\sqrt{N \pi}}{2 \sqrt{K}} \mathbf{I}_{K}\right)^{-1}\right) / K=\frac{4 K}{\pi N},
\end{aligned}
$$


where (a) comes from $\lim _{N \rightarrow \infty} \mathbf{F}_{\mathrm{RF}}^{(\mathrm{SP})^{\mathrm{H}}} \mathbf{F}_{\mathrm{RF}}^{(\mathrm{SP})}=\mathbf{I}_{K}$. Similar to (1), the achievable rate for each user depends on the received SNR which is $\rho / \Gamma^{(\mathrm{SP})}$ when ZF is applied at the baseband. Hence, the asymptotic achievable sum-rate is

$$
R^{(\mathrm{SP})} \rightarrow K \log _{2}\left(1+\frac{\pi \rho N}{4 K}\right) .
$$

Hybrid beamforming with phase shifter selection: By equipping each phase shifter with a dedicated ON/OFF switch, as shown in Fig. 1(b), phase shifter selection allows for identifying and turning off the phase shifters that have a negligible impact on spectral efficiency. Unlike the previous work [6]-[8], where phase shifter selection was performed according the singular vectors of the channel matrix, in the following $\mathbf{H}$ will be directly used to calculate the beamforming weights. Let $\operatorname{Pr}\left(H_{n, k}\right)$ denote the probability density function of $H_{n, k}$, and define a threshold level $\alpha$ to turn off the phase shifters. In this case, the $\mathrm{RF}$ beamformer is

$$
F_{n, k}^{(\text {SPSS })}= \begin{cases}\frac{\exp \left(j \angle H_{n, k}\right)}{\sqrt{L(1-\beta \%)}}, & \text { if } \alpha<\left|H_{n, k}\right|, \text { and } n \in \mathcal{I}_{k}, \\ 0, & \text { otherwise },\end{cases}
$$

where $\beta \%=\operatorname{Pr}\left(H_{n, k} \leq \alpha\right)$ is a measure of the reduction in the number of the active phase shifters. In the rest of the analysis, it is noted that $\alpha$ is chosen such that $K, L, N$ are integer numbers. When the number of antennas goes large, $\beta \%=1-\exp \left(-\alpha^{2}\right)$ since $\left|H_{n, k}\right|$ follows Rayleigh distribution [6].

To evaluate the performance of the proposed beamformer, let's define $h$ and $\tilde{h}$ where $h$ is a random variable with the same distribution as $H_{n, k}$, and

$$
\tilde{h}= \begin{cases}0 & \text { if }|h| \leq \alpha, \\ |h| & \text { if } \alpha<|h| .\end{cases}
$$

Then, similar to (3) for SP structure,

$$
\begin{aligned}
\hat{\mathbf{h}}_{k}^{\mathrm{H}} \mathbf{f}_{\mathrm{RF}, \mathrm{k}}^{\text {(SPS) }} & =\sum_{\forall n \in \mathcal{I}_{k}} \hat{H}_{n, k}^{*} F_{n, k}^{(\mathrm{SPSS})} \\
& =\sqrt{\frac{1}{K(1-\beta \%)}}\left(\frac{K \sum_{\forall n \in \mathcal{I}_{k}} \sqrt{N} \hat{H}_{n, k}^{*} F_{n, k}^{(\mathrm{SPSS})}}{N}\right) \\
& =\frac{\mathrm{E}[\tilde{h}]}{\sqrt{K(1-\beta \%)}}=\frac{\frac{\sqrt{\pi}}{2}+\alpha \mathrm{e}^{-\alpha^{2}}-\frac{\sqrt{\pi}}{2} \operatorname{erf}(\alpha)}{\sqrt{K(1-\beta \%)}}
\end{aligned}
$$

where $\mathrm{E}[\tilde{h}]$ for random variable $\tilde{h}$ was calculated in [6]. Following the same approach as in (4)-(5), the achievable sumrate using the beamformer in (6) becomes

$$
R^{(\mathrm{SPSS})} \rightarrow K \log _{2}\left(1+\frac{\pi \rho N\left(\frac{\sqrt{\pi}}{2}+\alpha \mathrm{e}^{-\alpha^{2}}-\frac{\sqrt{\pi}}{2} \operatorname{erf}(\alpha)\right)^{2}}{4 K(1-\beta \%)}\right) .
$$

Hybrid beamforming with reduced number of phase shifters: In this structure, as shown in Fig. 1(c), each phase shifter can be connected to only of the $S$ adjacent antennas. Hence, there will be only $N / S$ active antennas at each channel realization. Instead of designing the beamformer according to the thin-SVD

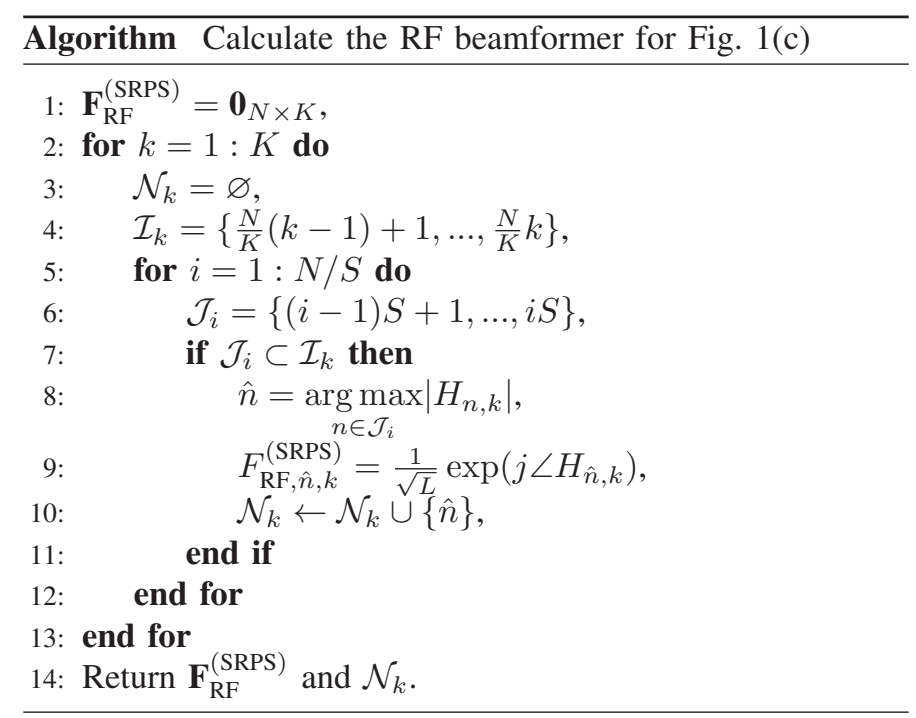

of $\mathbf{H}^{\mathrm{H}}$ [7], Algorithm 1 presents an alternative solution to calculate $\mathbf{F}_{\mathrm{RF}}^{\text {(SRPS) }}$ directly from $\mathbf{H}$. Let's use index $i \in\{1, \ldots, K L\}$ to label all phase shifters where $L=N / K S$. The $i$ th phase shifter selects antenna with index $\hat{n}$ from $\mathcal{J}_{i}=\{(i-1) S+1, \ldots, i S\}$ where $\hat{n}=\arg \max \left|H_{n, k}\right|$. Let $\mathcal{N}_{k}$ denote the set of the indexes of the active antennas that are connected to the $k$ th RF chain, and define $\breve{H}$ as a random variable that has the same distribution as $\max _{n \in \mathcal{J}_{i}}\left|H_{n, k}\right|$. In other words, $\breve{H}$ is the maximum of $S$ random variables with Normal distribution. Similar to the sum-rate calculation for the other structures, the performance depends on

$$
\begin{aligned}
\hat{\mathbf{h}}_{k}^{\mathrm{H}} \mathbf{f}_{\mathrm{RF}, \mathrm{k}}^{(\mathrm{SRPS})} & =\sum_{\forall n \in \mathcal{I}_{k}} \hat{H}_{n, k}^{*} F_{n, k}^{(\mathrm{SRPS})}=\frac{1}{\sqrt{L}} \sum_{n \in \mathcal{N}_{m}}\left|\hat{H}_{n, k}\right| \\
& =\frac{1}{L \sqrt{K S}} \sum_{n \in \mathcal{N}_{m}}\left|\sqrt{N} \hat{H}_{n, k}\right|=\frac{\mathrm{E}[\breve{H}]}{\sqrt{K S}} .
\end{aligned}
$$

The expected value of $\breve{H}$ was calculated in [7]. Finally, the achievable sum-rate is

$$
R_{\mathrm{RF}}^{(\mathrm{SRPS})}=K \log _{2}\left(1+\frac{\left(\sum_{s=0}^{S-1}\left(\begin{array}{c}
S-1 \\
s
\end{array}\right) \frac{(-1)^{s}}{(s+1)^{3 / 2}}\right)^{2} \rho N S \pi}{4 K}\right) .
$$

Remark 1. It is noted that the closed-forms derived in this section are very similar to that of [7] where the RF beamformers where calculated according to the singular vectors of $\mathbf{H}^{\mathrm{H}}$. The only difference with the closed-forms in [7] is that the new approach has factor $N$ inside the logarithms whereas it was $N-K$ in [7]. This difference in terms of spectral efficiency is negligible considering that $K \ll N$. However, in terms of computational complexity the new approach results in $O\left(K^{3}\right)$ instead of $O\left(K N^{2}\right)$.

In order to relax the perfect CSI assumption and to consider correlated Rayleigh fading channel, a quantized hybrid beamforming and channel estimation for the structures in Fig. 1 will be proposed in the next section. 


\section{QuANTIZED BEAMFORMING FOR CORRELATED RAYLEIGH FADING CHANNELS}

Traditionally, most of the beamforming algorithms are developed under perfect CSI assumption, and then they are applied to real world scenarios with channel estimation errors [14]. However, acquiring the estimate of the propagation channel matrix $\tilde{\mathbf{H}} \in \mathbb{C}^{N \times K}$ is a tedious task in massive MIMO systems with hybrid beamformers. This challenge applies to both frequency/time division duplex (FDD/TDD) operations. In an FDD system, the estimation time grows with $N$ for uncorrelated Rayleigh fading channels. For a TDD system, on the other hand, consider a scenario with a single-antenna user. In order for the base station to acquire $\tilde{\mathbf{H}}$ in uplink, at least $N / K$ measurements are needed which results in a large signaling overhead. Hence, it is necessary for our proposed hybrid beamformers to operate with less stringent constraints on the availability of CSI. Moreover, in practical systems, the base station is usually located on top of a tall building or tower, and it covers a sector that includes users which are surrounded by local scatterers. Hence, it is reasonable to consider a scenario that the channel is correlated at the base station side whereas the channel at the user side is uncorrelated [15]. In the following, it is assumed that the channel follows block fading model and it remains constant during the estimation and data transmission phases.

Let $\mathbf{h}_{k}=\mathbf{R}_{k}^{1 / 2} \mathbf{h}_{\mathrm{w}, k}$ represent the correlated channel vector for the $k$ th user where $\mathbf{R}_{k}$ denotes the correlation matrix, and $\mathbf{h}_{\mathrm{w}, k}$ follows i.i.d. uncorrelated Normal distribution. Using the Karhunen-Loeve representation, $\mathbf{h}_{k}$ can be written in the form of $\mathbf{h}_{k}=\mathbf{U}_{k} \boldsymbol{\Lambda}_{k}^{1 / 2} \mathbf{g}_{k}$ where $\mathbf{U} \in \mathbb{C}^{N \times r_{k}}$ is semi-unitary matrix of the eigenvectors, $\boldsymbol{\Lambda}_{k}$ is a diagonal matrix that contains the $r_{k}$ nonzero eigenvalues of $\mathbf{R}_{k}$, and the elements of $\mathbf{g}_{k} \in \mathbb{C}^{r_{k} \times 1}$ are i.i.d. and uncorrelated with Normal distribution. For notation simplicity and without loss of generality, let $r_{k}=r, \forall k \in$ $\{1, \ldots, K\}$. In addition, assume that the second order statistics of the channel is available at base station and there is a shared codebook $\mathcal{C}_{k}$ between the base station and user $k$.

Under correlated Rayleigh fading assumption, the channel vectors can be effectively quantized by $\mathbf{c}_{k, b} \in \mathcal{C}_{k}$ where [16], [17]

$$
\mathbf{c}_{k, b}=\frac{\mathbf{R}_{k}^{1 / 2} \mathbf{w}_{b}}{\left\|\mathbf{R}_{k}^{1 / 2} \mathbf{w}_{b}\right\|}, b \in\{1, \ldots, B\},
$$

and $B$ is the codebook size and $\mathbf{w}_{b}$ is a random unite-norm vector which its direction is uniformly distributed over an $N$-dimension hyper-sphere. To achieve a near-optimal performance, the codebook size for (12) grows with $r$ instead of $N$ for random vector quantization [16], [17]. This is significant improvement as $r$ is typically a small number in many real world scenarios [15]. Assuming that the base station has a fullydigital beamformer, $\mathbf{c}_{k, b}^{\star}$ can be used as an estimation of channel direction $\hat{\mathbf{h}}_{k}$ where

$$
\mathbf{c}_{k, b}^{\star}=\underset{\mathbf{c}_{k, b}}{\arg \max }\left|\mathbf{c}_{k, b}^{\mathrm{H}} \hat{\mathbf{h}}_{k}\right|^{2} \text {, subject to (s.t.) } \mathbf{c}_{k, b} \in \mathcal{C}_{k} .
$$

However, it is not possible to directly use (13) in hybrid beamforming due to the constraints that are imposed by switches and phase shifters. In the following, we will explain codebook generation, channel estimation and hybrid beamforming procedures for the structures in Fig. 1.

Codebook generation for user $k$ : Consider that user $k$ is the only existing user in the system. The codebook generation consists of two steps:

1) Create unconstrained codebook $\mathcal{C}_{k}$ according to (12). Consider each codeword $\mathbf{c}_{k, b} \forall b \in\{1, \ldots B\}$ of $\mathcal{C}_{k}$ as a realization of perfect channel vector for user $k$, i.e. assume $\hat{\mathbf{h}}_{k}=\mathbf{c}_{k, b}$.

2) For each $\mathbf{c}_{k, b}$, use the proposed hybrid beamforming methods in Section III to create a corresponding RF beamforming codeword $\mathbf{c}_{k, b}^{(q)}$. Finally, let $\mathcal{C}_{k}^{(q)}$ contain the set of vectors $\mathbf{c}_{k, b}^{(q)}, \forall b \in\{1, \ldots, B\}$ for each structure.

Proposed channel estimation and data transmission schemes for TDD operation: In the estimation phase, only one user at a time will transmit its pilots over $B$ transmission slots. At the $b$ th transmission, $\forall b \in\{1, \ldots, B\}$, the base station sets its RF beamformer according to $\mathbf{c}_{k, b}^{(q)}$ of the codebook $\mathcal{C}_{k}^{(q)}$. Then, it selects the codeword

$$
\mathbf{c}_{k}^{(q)^{\star}}=\underset{\mathbf{c}_{k, b}^{(q)}}{\arg \max }\left|\mathbf{c}_{k, b}^{(q)^{\mathrm{H}}} \hat{\mathbf{h}}_{k}\right|^{2} \text {, s.t. } \mathbf{c}_{k, b} \in \mathcal{C}_{k}^{(q)} .
$$

This procedure is repeated for the $K$ users which results in $K B$ transmissions. Then, the base station creates the beamforming matrix

$$
\mathbf{F}_{\mathrm{RF}}^{(q)^{\star}}=\left[\mathbf{c}_{1}^{(q)^{\star}}, \ldots, \mathbf{c}_{K}^{(q)^{\star}}\right] .
$$

The effective channel $\mathbf{H}_{\mathrm{e}}^{(q)} \in \mathbb{C}^{K \times K}$ at the baseband is $\mathbf{H}_{\mathrm{e}}^{(q)}=\mathbf{H}^{\mathrm{H}} \mathbf{F}_{\mathrm{RF}}^{(q)}$ which will be estimated in the next $K$ time slots by allowing each user to transmit a pilot at each instant. Hence, in total $(B+1) K$ transmissions are needed for CSI estimation. It is noted that any estimator, such as least squares or minimum mean square error, can be used in the last $K$ transmissions. We model an erroneous estimate of the effective channel, which is due to noise, as $\tilde{\mathbf{H}}_{\mathrm{e}}^{(q)}=\sqrt{1-e^{2}} \mathbf{H}_{\mathrm{e}}^{(q)}+e \mathbf{E}$ where $e$ is a measure of estimation error and $\mathbf{E} \in \mathbb{C}^{K \times K}$ is the error matrix with i.i.d. complex Normal distribution. Finally, the data transmission phase starts from the $K(B+1)+1$ transmission slot. At this stage, the RF beamformer is the same as (15) and the baseband precoder is set according to $\mathbf{F}_{\mathrm{B}}^{(q)}=\left(\mathbf{H}^{\mathrm{H}} \mathbf{F}_{\mathrm{RF}}^{(q)}\right)^{-1}$.

Remark 2. The proposed codebook and transmission methods can be applied in FDD transmission with minor modifications. For FDD operation two rounds of channel estimation and feedback are required. Firstly, the base station transmits pilots to each user by using the codebook $\mathcal{C}_{k}^{(q)}$. User $k$ selects the codeword index that satisfies (14) and transmits it to the base station with $\log _{2}(B)$ bits of feedback. Then, the base station sets its RF beamforming matrix according to the received indexes and (15). Finally, a second round of channel estimation and feedback is needed to estimate $\tilde{\mathbf{H}}_{\mathrm{e}} \in \mathbb{C}^{K \times K}$ at the base station. 


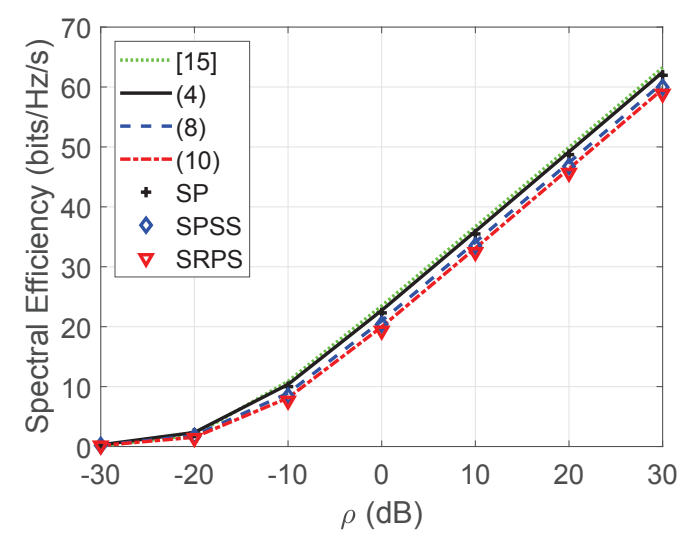

Fig. 2. Achievable sum-rates by simulations and closed-forms in (5), (9) and (11) for uncorrelated i.i.d. Rayleigh fading channel with perfect CSI. $N=128, K=4, \beta=75 \%$ and $S=4$.

\section{Simulation Results}

In this section, Monte Carlo simulations over 1000 realizations are used to evaluate the average performance of the proposed techniques. Under perfect CSI and uncorrelated Rayleigh fading assumption, Fig. 2 presents the spectral efficiency achieved by simulations and the closed-forms in (5), (9) and (11) for $N=256, K=4, \beta=75 \%$ and $S=4$. It is noted that $\beta=75 \%$ and $S=4$ are equivalent to having only $K L=N / 4=64$ active phase shifters. Figure 2 also provides a comparison with the successive interference cancellation based RF beamformer of [12]. It is observed that our method provides almost the same performance as [12] which has higher complexity as multiple computations of SVD and inversions of matrices with larger dimension than $K \times K$ are required. In addition to observing a good match between the simulations and closed-forms in (5), (9) and (11), Fig. 2 shows that the proposed phase shifter selection based structures effectively reduce the number of the active phase shifters while providing a comparable performance to that of the subconnected structures with $N$ active phase shifters.

Figure 3 presents the achievable sum-rates and energy efficiency (EE) of the proposed beamformers with phase shifter selection compared to that of digital ZF. The EE is defined as $E=$ $\frac{W R^{(q)}}{P_{\text {tot }}^{(q)}}$, where $W$ and $P_{\text {tot }}$ denote bandwidth and total amount of power that is required to achieve sum-rate $R$. To model $P_{\text {tot }}$, let $P_{\mathrm{tot}}=P+P_{\mathrm{Syn}}+N_{\mathrm{RF}} P_{\mathrm{RF}}+N_{\mathrm{PS}} P_{\mathrm{PS}}+N_{\mathrm{S}} P_{\mathrm{S}}$ where $P$, $P_{\text {Syn }}$ represent the transmit power and power consumption of the synthesizer. $N_{\mathrm{RF}}, N_{\mathrm{PS}}, N_{\mathrm{S}}, P_{\mathrm{RF}}, P_{\mathrm{PS}}$ and $P_{\mathrm{S}}$ denote the numbers and the power consumption of the RF chains, phase shifters and switches, respectively. The power consumption model for digital beamformer is $P_{\text {tot }}^{(\mathrm{D})}=P+P_{\mathrm{Syn}}+N P_{\mathrm{RF}}$, for $\mathrm{SP}$ is $P_{\mathrm{tot}}^{(\mathrm{SP})}=P+P_{\mathrm{Syn}}+K P_{\mathrm{RF}}+N_{\mathrm{PS}} P_{\mathrm{PS}}$, for SPSS is $P_{\text {tot }}^{(\text {SPSS })}=P+P_{\mathrm{Syn}}+K P_{\mathrm{RF}}+(1-\beta / 100) N P_{\mathrm{PS}}+N P_{\mathrm{S}}$ and for SRPS structure is $P_{\text {tot }}^{(\mathrm{SRPS})}=P+P_{\mathrm{Syn}}+K P_{\mathrm{RF}}+N P_{\mathrm{PS}} / S+N P_{\mathrm{S}}$ [8]. Similar to [18], we set $P_{\mathrm{RF}}=1 \mathrm{~W}, P_{\text {Syn }}=2 \mathrm{~W}$ and $W=20 \mathrm{MHz}$. The power consumption of each of the phase shifters $P_{\mathrm{PS}}$ and switches $P_{\mathrm{S}}$ at $2.4 \mathrm{GHz}$ are reported
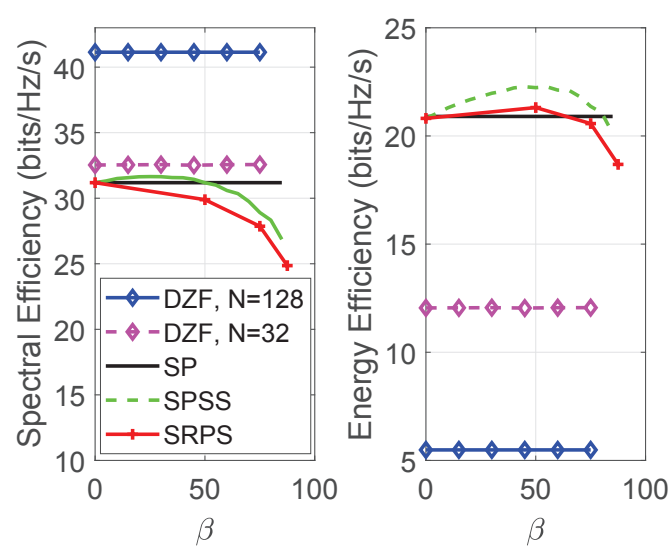

Fig. 3. Spectral efficiency (left) and EE (right) vs $\beta$ (left).

as $28.8 \leq P_{\mathrm{PS}} \leq 152 \mathrm{~mW}$ [19] and $0<P_{\mathrm{S}} \leq 15 \mathrm{~mW}$ [20]. In the following, we use $P=20 \mathrm{~W}, P_{\mathrm{PS}}=30 \mathrm{~mW}$, $P_{\mathrm{S}}=1 \mathrm{~mW}, \rho=10 \mathrm{~dB}$ and $K=4$. As a performance benchmark, in addition to fully-digital system with $N=128$, we consider $\mathrm{ZF}$ with $N / K=32$ antennas as the array gain of subconnected structures is related to $N / K$. It could be easily verified that setting $S \in\{1,2,4,8\}$ is equivalent to $\beta \in\{0 \%, 50 \%, 75 \%, 87.5 \%\}$. As expected, the fully-digital $\mathrm{ZF}$ with $N=128$ provides highest sum-rate, however it has the worst EE. The EE of hybrid beamformers, with 4 RF chains and 128 antennas, is much higher than fully-digital systems. They provide a comparable spectral efficiency compared to fullydigital $\mathrm{ZF}$ with $N=32 \mathrm{RF}$ chains.

To evaluate the performance of the quantized beamformers with respect to the codebook size, let $\rho=0 \mathrm{~dB}, e=0$, $r=4, \mathbf{U}_{k}$ be any tall semi-unitary random matrix, the diagonal elements of $\boldsymbol{\Lambda}_{k}$ be simply chosen according to uniform distribution distribution over [0,1] , and $\mathbf{R}_{k}$ be normalized such that $\operatorname{trace}\left(\mathbf{R}_{k}\right)=N$. The left plot in Fig. 4 shows that increasing the codebook size $B$ does not have a significant impact on the sumrates, however, it will increase the channel estimation overhead. On the other hand, the right plot in Fig. 4 demonstrates that, for $B=16, S=2, \beta=50 \%$, increasing $N$ provides performance gains. This result is significant as it allows for scaling up the array size just by using simple switches, yet the channel estimation overhead is fixed and the spectral efficiency is increasing. In addition, the computational complexity of the beamformer design is only limited to $O\left(K^{3}\right)$.

Finally, Fig. 5 considers the impact of estimation error $e$ on spectral efficiency. For the sake of comparison, consider the scenario where a huge signaling overhead is afforded to estimate the propagation channel matrix $\tilde{\mathbf{H}}=\sqrt{1-e^{2}} \mathbf{H}+e \mathbf{E}$. Using the traditional approach, the methods of section III are used to design the beamformers as if $\tilde{\mathbf{H}}$ is perfect CSI. Compared to proposed techniques, Fig. 5 shows that the quantized beamformers outperform the traditional approach when the same error variance $e=0.1$ is used in both scenarios. This result indicates that even if the channel was constant over a long period and estimating $\tilde{\mathbf{H}}$ was feasible, it is still beneficial 

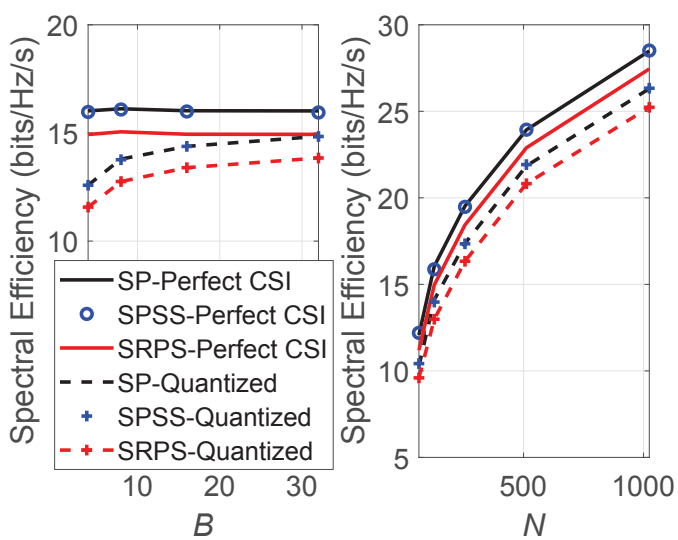

Fig. 4. Spectral efficiency vs $B$ (left) with $N=128 . R^{(q)}$ vs $N$ with $B=16$ (right), $K=4, \beta=50 \%, S=2, \rho=0 \mathrm{~dB}, e=0$ and $r=4$.

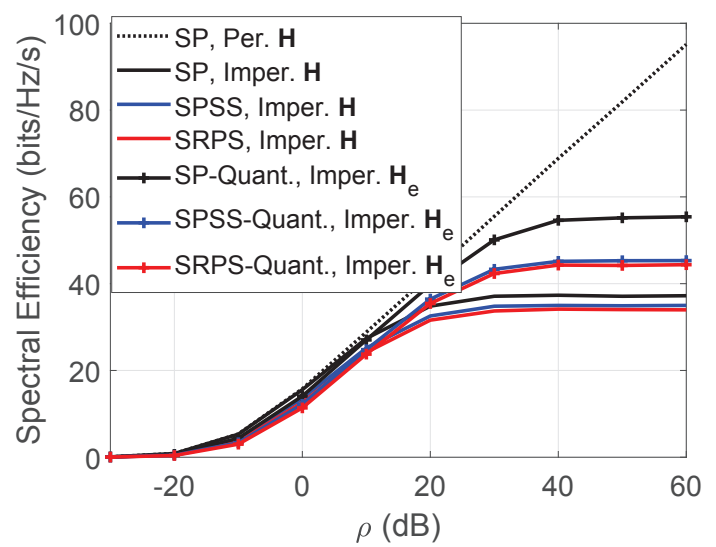

Fig. 5. Spectral efficiency vs $\rho . N=128, K=4, \beta=75 \%, S=4$, $e=0.1, B=16$ and $r=4$.

to use the quantized beamformers and only estimate $\tilde{\mathbf{H}}_{\mathrm{e}}^{(q)}$.

\section{CONCLUSION}

In this paper, we showed that hybrid beamforming is a promising approach to reduce the computational complexity of beamformers in massive MIMO systems to $O\left(K^{3}\right)$. In this scheme, the RF beamformer is directly inferred from the channel matrix and $O\left(K^{3}\right)$ only belongs to the baseband precoder. In addition, it allows for scaling up the array size at the base station by simply adding low-cost antennas and switches. The presented closed-forms can be used as a guide to evaluate the behavior of the hybrid beamformers with switches and phase shifters. We also developed codebook-based hybrid beamforming and channel estimation methods for correlated Rayleigh fading scenario. The channel estimation overhead increases with the rank of the correlation matrix instead of the number of the base station antennas. Finally, the proposed beamformers are applicable to both TDD and FDD massive MIMO systems.

\section{REFERENCES}

[1] F. Rusek, D. Persson, B. K. Lau, E. Larsson, T. Marzetta, O. Edfors, and F. Tufvesson, "Scaling up MIMO: Opportunities and challenges with very large arrays," Signal Processing Magazine, IEEE, vol. 30, no. 1, pp. 40-60, January 2013.

[2] S. Payami and F. Tufvesson, "Delay spread properties in a measured massive MIMO system at $2.6 \mathrm{GHz}$," IEEE Annual International Symposium on Personal, Indoor, and Mobile Radio Communications (PIMRC), pp. 53-57, Sept 2013.

[3] _ - "Channel measurements and analysis for very large array systems at $2.6 \mathrm{GHz}$," IEEE European Conference on Antennas and Propagation (EUCAP), pp. 433-437, March 2012.

[4] C. G. Tsinos, S. Maleki, S. Chatzinotas, and B. Ottersten, "On the energyefficiency of hybrid analog-digital transceivers for single- and multicarrier large antenna array systems," IEEE Journal on Selected Areas in Communications, vol. 35, no. 9, pp. 1980-1995, Sept 2017.

[5] S. Payami, M. Ghoraishi, and M. Dianati, "Hybrid beamforming for downlink massive mimo systems with multiantenna user equipment," IEEE Vehicular Technology Conference (VTC-Fall), pp. 1-5, Sept 2017.

[6] _ - "Hybrid beamforming for large antenna arrays with phase shifter selection," IEEE Transactions on Wireless Communications, vol. 15, no. 11, pp. 7258-7271, Nov 2016.

[7] S. Payami, M. Ghoraishi, M. Dianati, and M. Sellathurai, "Hybrid beamforming with reduced number of phase shifters for massive MIMO systems," IEEE Transactions on Vehicular Technology, vol. PP, no. 99, pp. 1-1, 2018.

[8] S. Payami, , N. M. Balasubramanya, C. Masouros, and M. Sellathurai, "An energy efficiency perspective on hybrid beamforming with phase shifters and switches," Submitted to IEEE Wireless Communication Letters.

[9] S. Payami, M. Shariat, M. Ghoraishi, and M. Dianati, "Effective RF codebook design and channel estimation for millimeter wave communication systems," IEEE International Conference on Communication Workshop (ICCW), pp. 1226-1231, June 2015.

[10] X. Zhang, A. Molisch, and S.-Y. Kung, "Variable-phase-shift-based RFbaseband codesign for MIMO antenna selection," IEEE Transactions on Signal Processing, vol. 53, no. 11, pp. 4091-4103, November 2005.

[11] O. El Ayach, S. Rajagopal, S. Abu-Surra, Z. Pi, and R. Heath, "Spatially sparse precoding in millimeter wave MIMO systems," IEEE Transactions on Wireless Communications, vol. 13, no. 3, pp. 1499-1513, March 2014.

[12] X. Gao, L. Dai, S. Han, C. L. I, and R. W. Heath, "Energy-efficient hybrid analog and digital precoding for MmWave MIMO systems with large antenna arrays," IEEE Journal on Selected Areas in Communications, vol. 34, no. 4, pp. 998-1009, April 2016.

[13] L. Liang, W. Xu, and X. Dong, "Low-complexity hybrid precoding in massive multiuser MIMO systems," IEEE Wireless Communications Letters, vol. 3, no. 6, pp. 653-656, Dec 2014.

[14] B. Clerckx, H. Joudeh, C. Hao, M. Dai, and B. Rassouli, "Rate splitting for MIMO wireless networks: a promising PHY-layer strategy for LTE evolution," IEEE Communications Magazine, vol. 54, no. 5, pp. 98-105, May 2016.

[15] A. Adhikary, J. Nam, J. Y. Ahn, and G. Caire, "Joint spatial division and multiplexing - the large-scale array regime," IEEE Transactions on Information Theory, vol. 59, no. 10, pp. 6441-6463, Oct 2013.

[16] W. Shen, L. Dai, Y. Zhang, J. Li, and Z. Wang, "On the performance of channel-statistics-based codebook for massive MIMO channel feedback," IEEE Transactions on Vehicular Technology, vol. 66, no. 8, pp. 75537557, Aug 2017.

[17] D. J. Love and R. W. Heath, "Limited feedback diversity techniques for correlated channels," IEEE Transactions on Vehicular Technology, vol. 55, no. 2, pp. 718-722, March 2006.

[18] A. Garcia-Rodriguez, V. Venkateswaran, P. Rulikowski, and C. Masouros, "Hybrid analog-digital precoding revisited under realistic RF modeling," IEEE Wireless Communications Letters, vol. 5, no. 5, pp. 528-531, Oct 2016.

[19] Y. Zheng and C. E. Saavedra, "An ultra-compact CMOS variable phase shifter for 2.4-GHz ISM applications," IEEE Transactions on Microwave Theory and Techniques, vol. 56, no. 6, pp. 1349-1354, June 2008.

[20] N. A. Talwalkar, C. P. Yue, H. Gan, and S. S. Wong, "Integrated CMOS transmit-receive switch using LC-tuned substrate bias for 2.4-GHz and 5.2-GHz applications," IEEE Journal of Solid-State Circuits, vol. 39, no. 6, pp. 863-870, June 2004. 